\title{
Asymmetric 1,4-Addition with a Chiral Calcium-Pybox Catalyst
}

\section{Gategory}

Polymer-Supported Synthesis

\section{Key words}

asymmetric catalysis

Michael addition

calcium

continuous-flow synthesis

\section{SYNFACGDinh}

Flow conditions

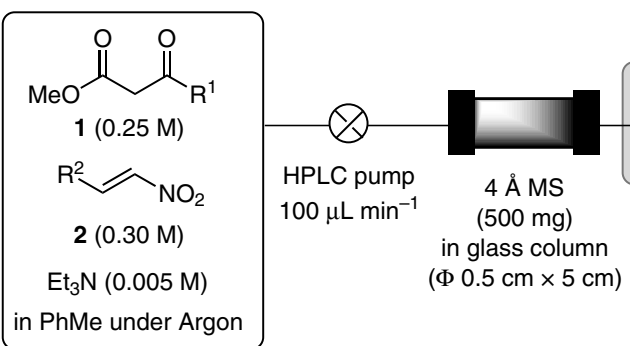

Selected examples:<smiles>COC(=O)C(C(=O)OC)C(C[N+](=O)[O-])c1ccccc1</smiles>

3a $(12-216 \mathrm{~h})$ $88-98 \%$ yield, $91-93 \%$ ee TON $=228$<smiles>COC(=O)C([C](C[N+](=O)[O-])c1ccc(F)cc1)C(=O)OC</smiles>
$94-95 \%$ yield, $90 \%$ ee<smiles>COC(=O)C([C](C[N+](=O)[O-])c1ccc(C)cc1)C(=O)OC</smiles>

3b $(12-18 \mathrm{~h})$ 90-92\% yield, $91 \%$ ee<smiles>COC(=O)C(C(=O)OC)[C@H](C[N+](=O)[O-])c1ccco1</smiles>

3f $(12-18 \mathrm{~h})$ $76-82 \%$ yield, $91 \%$ ee constant-temperature bath, $0{ }^{\circ} \mathrm{C}$

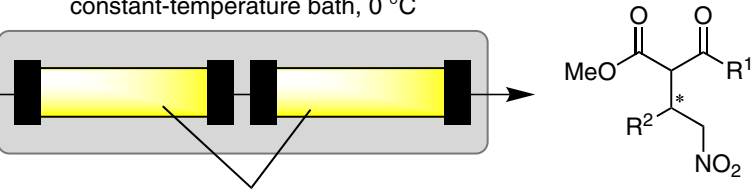

PS-Pybox $(750 \mathrm{mg} \times 2)$

$\mathrm{CaCl}_{2} \cdot 2 \mathrm{H}_{2} \mathrm{O}(375 \mathrm{mg} \times 2)$

in glass columns $(\Phi 1.0 \mathrm{~cm} \times 10 \mathrm{~cm})$<smiles>COC(=O)C([C](C[N+](=O)[O-])c1cccc(C)c1)C(=O)OC</smiles>

$3 c(12-18 \mathrm{~h})$ $81-88 \%$ yield, $95 \%$ ee<smiles>COC(=O)C(C(=O)OC)[C@H](C[N+](=O)[O-])C1CCCCC1</smiles>

$3 g(12-18 \mathrm{~h})$ $31-32 \%$ yield, $75-80 \%$ ee<smiles>COC(=O)C(Cc1ccc(OC)cc1)C(C(C)=O)C(=O)[O-]</smiles>

3d (12-18 h) $86-87 \%$ yield, $91 \%$ ee<smiles>COC(=O)C(C(C)=O)C(C[N+](=O)[O-])c1ccccc1</smiles>

3h (12-18 h) 95-96\% yield, $87-88 \%$ ee
Significance: A polymer-supported homochiral Pybox-calcium chloride complex catalyzed the asymmetric 1,4-addition of 1,3-dicarbonyl compounds $\mathbf{1}$ to nitroalkenes $\mathbf{2}$, to afford the corresponding adducts $\mathbf{3}$ in up to $98 \%$ yield and $95 \%$ ee under batch or flow conditions.
Comment: The flow system worked for 204 hours without significant loss of catalytic activity to give 3a in $95.5 \%$ yield with $92.0 \%$ ee on average. The total amount of product was $291.4 \mathrm{mmol}$ and the turn-over number (TON) reached 228.

sYNFACTS Contributors: Yasuhiro Uozumi, Yoichi M. A. Yamada, Aya Ohno Synfacts 2013,9(1), 0109 Published online: 17.12.2012 Dol: 10.1055/s-0032-1317727; Reg-No.: Y14412SF 